\title{
Statistics of boundary encounters by a particle diffusing outside a compact planar domain
}

\author{
Denis S. Grebenkov \\ E-mail: denis.grebenkov@polytechnique.edu \\ Laboratoire de Physique de la Matière Condensée (UMR 7643), \\ CNRS - Ecole Polytechnique, IP Paris, 91128 Palaiseau, France
}

\begin{abstract}
We consider a particle diffusing outside a compact planar set and investigate its boundary local time $\ell_{t}$, i.e., the rescaled number of encounters between the particle and the boundary up to time $t$. In the case of a disk, this is also the (rescaled) number of encounters of two diffusing circular particles in the plane. For that case, we derive explicit integral representations for the probability density of the boundary local time $\ell_{t}$ and for the probability density of the first-crossing time of a given threshold by $\ell_{t}$. The latter density is shown to exhibit a very slow long-time decay due to extremely long diffusive excursions between encounters. We briefly discuss some practical consequences of this behavior for applications in chemical physics and biology.

PACS numbers: 02.50.-r, 05.40.-a, 02.70.Rr, 05.10.Gg

Keywords: Boundary local time; Reflected Brownian motion; Diffusion-influenced reactions; Surface reactivity; Robin boundary condition; Heterogeneous catalysis
\end{abstract}

Submitted to: J. Phys. A: Math. Gen. 


\section{Introduction}

When a Brownian particle diffuses in a geometric confinement, its encounters with the reflecting boundary can be characterized by the boundary local time $\ell_{t}$, which plays the central role in the theory of stochastic processes [1-3]. In the basic setting of ordinary diffusion, one considers reflected Brownian motion $\boldsymbol{X}_{t}$, released at time $t=0$ from a fixed point $\boldsymbol{x}_{0}$ and diffusing inside an Euclidean domain $\Omega \subset \mathbb{R}^{d}$ with diffusion coefficient $D$ and normal reflections on the smooth boundary $\partial \Omega$. The boundary local time $\ell_{t}$ of this process on a subset of the boundary, $\Gamma \subset \partial \Omega$, is defined as

$$
\ell_{t}=\lim _{a \rightarrow 0} \frac{D}{a} \underbrace{\int_{0}^{t} d t^{\prime} \Theta\left(a-\left|\boldsymbol{X}_{t^{\prime}}-\Gamma\right|\right)}_{\text {residence time }},
$$

where $\Theta(z)$ is the Heaviside step function, and $|\boldsymbol{x}-\Gamma|$ denotes the distance between a point $\boldsymbol{x}$ and the boundary region $\Gamma$. In this definition, the integral is the residence time of reflected Brownian motion $\boldsymbol{X}_{t}$ up to time $t$ inside a thin boundary layer of width $a$ around $\Gamma: \Gamma_{a}=\{\boldsymbol{x} \in \Omega:|\boldsymbol{x}-\Gamma|<a\}$ (see [4-17] and references therein). In the limit $a \rightarrow 0$, the residence time vanishes but its rescaling by $a$ yields a well-defined nontrivial limit $\ell_{t}$. According to Eq. (1), the boundary local time $\ell_{t}$ is a non-decreasing process that remains constant when $\boldsymbol{X}_{t}$ is the bulk, and increases only when $\boldsymbol{X}_{t}$ hits the boundary. Alternatively, the boundary local time $\ell_{t}$ can also be written as

$$
\ell_{t}=\lim _{a \rightarrow 0} a \mathcal{N}_{t}^{a}
$$

where $\mathcal{N}_{t}^{a}$ is the number of downcrossings of the thin boundary layer $\Gamma_{a}$ up to time $t$ (Fig. 1), i.e., a regularized version of the number of encounters of the process with the region $\Gamma$ (see [18] for further discussion). For a fixed time $t, \ell_{t}$ is a random variable, which can be characterized by the probability density $\rho\left(\ell, t \mid \boldsymbol{x}_{0}\right)$. Note that $\ell_{t}$ has units of length, whereas $\ell_{t} / D$ has units of time per length, reflecting the rescaling by $a$ in Eq. (1).

While general stochastic properties of the boundary local time were thoroughly investigated in the past (see [20-22] and references therein), its geometry-specific properties are less known. For instance, how does the distribution of the number of encounters depend on the shape of the confining domain and evolve with time? When does this number exceed a prescribed threshold? To answer these questions, general spectral expansions for $\rho\left(\ell, t \mid \boldsymbol{x}_{0}\right)$ and for the probability density $U\left(\ell, t \mid \boldsymbol{x}_{0}\right)$ of the firstcrossing time $\mathcal{T}_{\ell}$ of a prescribed threshold $\ell$ by the process $\ell_{t}$, were established in $[18,23]$. These spectral expansions rely on the eigenmodes of the Dirichlet-to-Neumann operator, which are known explicitly only for some simple domains [24]. In particular, closed analytical formulas were derived for diffusion in a half-line,

$$
\rho\left(\ell, t \mid x_{0}\right)=\operatorname{erf}\left(\frac{x_{0}}{\sqrt{4 D t}}\right) \delta(\ell)+\frac{\exp \left(-\frac{\left(x_{0}+\ell\right)^{2}}{4 D t}\right)}{\sqrt{\pi D t}},
$$




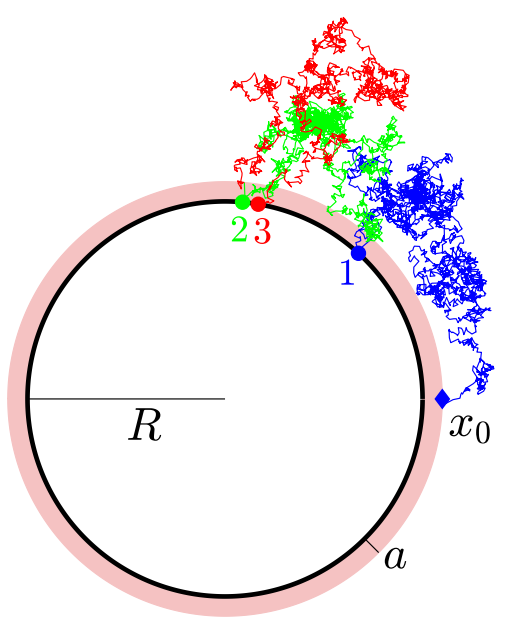

Figure 1. A simulated trajectory of reflected Brownian motion in the exterior of a disk of radius $R: \Omega=\left\{\boldsymbol{x} \in \mathbb{R}^{2}:|\boldsymbol{x}|>R\right\}$. Pink region denotes a thin boundary layer $\Gamma_{a}$ of width $a$ near the boundary: $\Gamma=\partial \Omega$. The particle is released in a close vicinity of the boundary (blue diamond) and diffuses until the first encounter with that boundary (filled circle enumerated by 1). From that point, the particle is released a distance $a$ above the circle and resumes its motion until the next encounter, and so on. Such a regularization with small $a>0$ allows one to define the number of encounters $\mathcal{N}_{t}^{a}$ and to split the trajectory into excursions between encounters (such three excursions are drawn by different colors).

$$
U\left(\ell, t \mid x_{0}\right)=\left(\ell+x_{0}\right) \frac{e^{-\left(\ell+x_{0}\right)^{2} /(4 D t)}}{\sqrt{4 \pi D t^{3}}},
$$

and in the exterior of a ball of radius $R$ :

$$
\begin{aligned}
\rho\left(\ell, t \mid \boldsymbol{x}_{0}\right) & =\left(1-\frac{R}{\left|\boldsymbol{x}_{0}\right|} \operatorname{erfc}\left(\frac{\left|\boldsymbol{x}_{0}\right|-R}{\sqrt{4 D t}}\right)\right) \delta(\ell) \\
& +\frac{e^{-\ell / R}}{\left|\boldsymbol{x}_{0}\right|}\left(\operatorname{erf}\left(\frac{\left|\boldsymbol{x}_{0}\right|-R+\ell}{\sqrt{4 D t}}\right)+\frac{R e^{-\left(\left|\boldsymbol{x}_{0}\right|-R+\ell\right)^{2} /(4 D t)}}{\sqrt{\pi D t}}\right), \\
U\left(\ell, t \mid \boldsymbol{x}_{0}\right) & =\frac{R e^{-\ell / R}}{\left|\boldsymbol{x}_{0}\right|} \frac{\left|\boldsymbol{x}_{0}\right|-R+\ell}{\sqrt{4 \pi D t^{3}}} e^{-\left(\left|\boldsymbol{x}_{0}\right|-R+\ell\right)^{2} /(4 D t)}
\end{aligned}
$$

where $\delta(\ell)$ is the Dirac distribution (see [24,25] for details).

In turn, the analysis of diffusion in the exterior of a compact planar domain turns out to be more subtle. As planar diffusion is recurrent, the diffusing particle never escapes to infinity and repeatedly returns to the boundary so that the boundary local time $\ell_{t}$ grows to infinity as $t \rightarrow \infty$ and thus crosses any threshold with probability 1. However, the probability of not hitting the boundary up to time $t$ is known to decay logarithmically slowly with $t$ [26], so that each return to the boundary may take abnormally long time. In this paper, we focus on diffusion outside a disk and derive explicit integral representations for both probability densities $\rho\left(\ell, t \mid \boldsymbol{x}_{0}\right)$ and $U\left(\ell, t \mid \boldsymbol{x}_{0}\right)$. We use then these presentations to analyze their asymptotic behavior. We also characterize the relative contributions of random trajectories with different exploration 
Statistics of boundary encounters...

sizes. Finally, we discuss extensions to more general planar domains, describe some applications in physics and chemistry, and outline several open questions.

\section{Formal solution}

In this Section, we recall the formal general solution for $\rho\left(\ell, t \mid \boldsymbol{x}_{0}\right)$ and $U\left(\ell, t \mid \boldsymbol{x}_{0}\right)$ from $[18,23]$. The central relation is the expression for the moment-generating function of the boundary local time $\ell_{t}$ :

$$
\mathbb{E}_{\boldsymbol{x}_{0}}\left\{e^{-q \ell_{t}}\right\}=S_{q}\left(t \mid \boldsymbol{x}_{0}\right),
$$

where $S_{q}\left(t \mid \boldsymbol{x}_{0}\right)$ is the survival probability, which satisfies the (backward) diffusion equation

$$
\partial_{t} S_{q}\left(t \mid \boldsymbol{x}_{0}\right)=D \Delta S_{q}\left(t \mid \boldsymbol{x}_{0}\right) \quad\left(\boldsymbol{x}_{0} \in \Omega\right),
$$

with the initial condition $S_{q}\left(0 \mid \boldsymbol{x}_{0}\right)=1$ and the mixed Robin-Neumann boundary condition:

$$
\begin{aligned}
& \left.\left(\partial_{n}+q\right) S_{q}\left(t \mid \boldsymbol{x}_{0}\right)\right|_{\Gamma}=0, \\
& \left.\partial_{n} S_{q}\left(t \mid \boldsymbol{x}_{0}\right)\right|_{\partial \Omega \backslash \Gamma}=0
\end{aligned}
$$

(for unbounded domains, the regularity condition $S_{q}\left(t \mid \boldsymbol{x}_{0}\right) \rightarrow 1$ as $\left|\boldsymbol{x}_{0}\right| \rightarrow \infty$ is also imposed). Here $\Delta$ is the Laplace operator acting on $\boldsymbol{x}_{0}, \partial_{n}$ is the normal derivative at the boundary oriented outward the domain, and $q \geq 0$ is a parameter in Eq. (7), which can be related to the surface reactivity of the subset $\Gamma$ [18]. On the other hand, the moment-generating function is defined via the probability density of $\ell_{t}$ :

$$
\mathbb{E}_{\boldsymbol{x}_{0}}\left\{e^{-q \ell_{t}}\right\}=\int_{0}^{\infty} d \ell e^{-q \ell} \rho\left(\ell, t \mid \boldsymbol{x}_{0}\right) .
$$

Inverting this Laplace transform, one formally gets

$$
\rho\left(\ell, t \mid \boldsymbol{x}_{0}\right)=\mathcal{L}_{q, \ell}^{-1}\left\{S_{q}\left(t \mid \boldsymbol{x}_{0}\right)\right\} .
$$

The first-crossing time $\mathcal{T}_{\ell}$ of a given threshold $\ell \geq 0$ by the boundary local time $\ell_{t}$ is defined as

$$
\mathcal{T}_{\ell}=\inf \left\{t>0: \ell_{t}>\ell\right\}
$$

As the boundary local time is a non-decreasing process, the cumulative distribution function of the first-crossing time is determined as

$$
Q\left(\ell, t \mid \boldsymbol{x}_{0}\right)=\mathbb{P}_{\boldsymbol{x}_{0}}\left\{\mathcal{T}_{\ell}<t\right\}=\mathbb{P}_{\boldsymbol{x}_{0}}\left\{\ell_{t}>\ell\right\}=1-\mathcal{L}_{q, \ell}^{-1}\left\{S_{q}\left(t \mid \boldsymbol{x}_{0}\right) / q\right\},
$$

where we used Eq. (12). The probability density of the first-crossing time follows:

$$
U\left(\ell, t \mid \boldsymbol{x}_{0}\right)=\partial_{t} Q\left(\ell, t \mid \boldsymbol{x}_{0}\right)=\mathcal{L}_{q, \ell}^{-1}\left\{\frac{-\partial_{t} S_{q}\left(t \mid \boldsymbol{x}_{0}\right)}{q}\right\} .
$$


Statistics of boundary encounters...

Recalling that $H_{q}\left(t \mid \boldsymbol{x}_{0}\right)=-\partial_{t} S_{q}\left(t \mid \boldsymbol{x}_{0}\right)$ is the probability density of the first-passage time to a partially reactive target $\Gamma$ (see, e.g., [26]), one can rewrite Eq. (15) as

$$
H_{q}\left(t \mid \boldsymbol{x}_{0}\right)=\int_{0}^{\infty} d \ell q e^{-q \ell} U\left(\ell, t \mid \boldsymbol{x}_{0}\right) .
$$

In other words, the probability density $U\left(\ell, t \mid \boldsymbol{x}_{0}\right)$ determines the whole family of the first-passage times described by $H_{q}\left(t \mid \boldsymbol{x}_{0}\right)$ [18].

Even though the probability densities $\rho\left(\ell, t \mid \boldsymbol{x}_{0}\right)$ and $U\left(\ell, t \mid \boldsymbol{x}_{0}\right)$ are formally determined via Eqs. $(12,15)$, their analysis requires the Laplace transform inversion, $\mathcal{L}_{q, \ell}^{-1}$, which may be numerically unstable [27]. Moreover, as the parameter $q$ enters through the Robin boundary condition (9), the dependence of the survival probability $S_{q}\left(t \mid \boldsymbol{x}_{0}\right)$ on $q$ is implicit that renders its analysis even more difficult. The aim of the present paper is to derive fully explicit representations for both densities in the case of diffusion outside a disk and then to use them for the asymptotic analysis.

\section{Diffusion outside a disk}

In this section, we consider reflected Brownian motion in the exterior of the disk of radius $R, \Omega=\left\{\boldsymbol{x} \in \mathbb{R}^{2}:|\boldsymbol{x}|>R\right\}$, and focus on the boundary local time $\ell_{t}$ on the boundary of that disk: $\Gamma=\partial \Omega$ (Fig. 1). The rotational invariance of the domain implies that the probability densities and related quantities do not depend on the angular coordinate. In the following, we replace the starting point $\boldsymbol{x}_{0}$ by its radial coordinate $r_{0}=\left|\boldsymbol{x}_{0}\right|$.

We start by recalling the integral representation for the survival probability $S_{q}\left(t \mid r_{0}\right)$, which can be derived from the classical solution of a similar heat conduction problem $[28,29]$

$$
\begin{aligned}
S_{q}\left(t \mid r_{0}\right) & =\frac{2 q R}{\pi} \int_{0}^{\infty} \frac{d z}{z} e^{-z^{2} D t / R^{2}} \\
& \times \frac{Y_{0}\left(z r_{0} / R\right)\left(z J_{1}(z)+q R J_{0}(z)\right)-J_{0}\left(z r_{0} / R\right)\left(z Y_{1}(z)+q R Y_{0}(z)\right)}{\left(z J_{1}(z)+q R J_{0}(z)\right)^{2}+\left(z Y_{1}(z)+q R Y_{0}(z)\right)^{2}},
\end{aligned}
$$

where $J_{\nu}(z)$ and $Y_{\nu}(z)$ are the Bessel functions of the first and second kind, respectively. To render the dependence of this expression on $q$ even more explicit, we represent it as

$$
S_{q}\left(t \mid r_{0}\right)=\frac{4}{\pi} \int_{0}^{\infty} \frac{d z}{z} e^{-z^{2} D t / R^{2}} \operatorname{Re}\left(\frac{q A\left(z, r_{0} / R\right)}{B(z) / R+q}\right),
$$

where

$$
\begin{aligned}
& A(z, r)=\frac{i}{2} \frac{\left(J_{0}(z)+i Y_{0}(z)\right)\left(J_{0}(z r)-i Y_{0}(z r)\right)}{J_{0}^{2}(z)+Y_{0}^{2}(z)} \\
& B(z)=\frac{z\left(J_{0}(z) J_{1}(z)+Y_{0}(z) Y_{1}(z)\right)+i \frac{2}{\pi}}{J_{0}^{2}(z)+Y_{0}^{2}(z)} .
\end{aligned}
$$


Statistics of boundary encounters...

Substituting Eq. (18) into Eq. (12) and evaluating the inverse Laplace transform with respect to $q$, we get

$$
\begin{aligned}
\rho\left(\ell, t \mid r_{0}\right) & =S_{\infty}\left(t \mid r_{0}\right) \delta(\ell) \\
& -\frac{4}{\pi R} \int_{0}^{\infty} \frac{d z}{z} e^{-z^{2} D t / R^{2}} \operatorname{Re}\left(A\left(z, r_{0} / R\right) B(z) e^{-B(z) \ell / R}\right),
\end{aligned}
$$

where

$$
S_{\infty}\left(t \mid r_{0}\right)=\frac{4}{\pi} \int_{0}^{\infty} \frac{d z}{z} e^{-z^{2} D t / R^{2}} \operatorname{Re}\left(A\left(z, r_{0} / R\right)\right)
$$

is obtained as the limit of Eq. (18) when $q \rightarrow \infty$. As in Eqs. (3, 5), the first term in Eq. (21) accounts for trajectories that did not hit the boundary up to time $t$, for which the boundary local time $\ell_{t}$ remained 0 . The positive-order moments of the boundary local time are analyzed in Appendix A. Similarly, Eq. (14) implies that the inverse Laplace transform of $S_{q}\left(t \mid r_{0}\right) / q$ with respect to $q$ yields

$$
Q\left(\ell, t \mid r_{0}\right)=1-\frac{4}{\pi} \int_{0}^{\infty} \frac{d z}{z} e^{-z^{2} D t / R^{2}} \operatorname{Re}\left(A\left(z, r_{0} / R\right) e^{-B(z) \ell / R}\right) .
$$

The time derivative of this expression gives the probability density $U\left(\ell, t \mid r_{0}\right)$ of the first-crossing time:

$$
U\left(\ell, t \mid r_{0}\right)=\frac{4 D}{\pi R^{2}} \int_{0}^{\infty} d z z e^{-z^{2} D t / R^{2}} \operatorname{Re}\left(A\left(z, r_{0} / R\right) e^{-B(z) \ell / R}\right) .
$$

Note also that setting $\ell=0$ in Eq. (24) yields

$$
U\left(0, t \mid r_{0}\right)=\frac{4 D}{\pi R^{2}} \int_{0}^{\infty} d z z e^{-z^{2} D t / R^{2}} \operatorname{Re}\left(A\left(z, r_{0} / R\right)\right)=H_{\infty}\left(t \mid r_{0}\right),
$$

where we used Eq. (22) and thus retrieved the probability density of the first-passage time to the disk.

The explicit integral representations $(21,23,24)$ are the main analytical results of the paper. Thanks to the exponential factor $e^{-z^{2} D t / R^{2}}$, these integrals rapidly converge for large $z$. In turn, the integrals in Eqs. $(21,23)$ exhibit logarithmically slow convergence at small $z$. A practical solution of this issue is discussed in Appendix B.

The boundary local time $\ell_{t}$ remains zero until the first encounter with the boundary. As a consequence, the first-crossing time $\mathcal{T}_{\ell}$ can be decomposed into two contributions: the first-passage time from $\boldsymbol{x}_{0}$ to the circle, $\mathcal{T}_{0, r_{0}}=\inf \left\{t>0: \ell_{t}>0|| \boldsymbol{X}_{0} \mid=r_{0}\right\}$, and the first-crossing time of the level $\ell$ after starting from the circle, $\mathcal{T}_{\ell, R}=\inf \{t>$ $\left.0: \ell_{t}>\ell|| \boldsymbol{X}_{0} \mid=R\right\}$ :

$$
\mathcal{T}_{\ell}=\mathcal{T}_{0, r_{0}}+\mathcal{T}_{\ell, R}
$$

The strong Markovian character of reflected Brownian motion and of the boundary local time, as well as the rotational invariance of the problem imply that these two 
Statistics of boundary encounters...

contributions are independent variables. As a consequence, the probability density of $\mathcal{T}_{\ell}$ can be obtained by convolving the densities of $\mathcal{T}_{0, r_{0}}$ and $\mathcal{T}_{\ell, R}$. The probability density of the first-passage time to the circle, $H_{\infty}\left(t \mid r_{0}\right)$, has been studied long ago (see $[26,29,30]$ and references therein). As a consequence, one can focus on the probability density $U(\ell, t \mid R)$ of the second contribution $\mathcal{T}_{\ell, R}$, for which $A(z, 1)=i / 2$, and Eq. (24) becomes

$$
U(\ell, t \mid R)=-\frac{2 D}{\pi R^{2}} \int_{0}^{\infty} d z z e^{-z^{2} D t / R^{2}} \operatorname{Im}\left(e^{-B(z) \ell / R}\right) .
$$

In the next section, we discuss the asymptotic behavior of the density $U\left(\ell, t \mid r_{0}\right)$.

\section{Asymptotic analysis}

To investigate the asymptotic behavior of the probability density $U\left(\ell, t \mid r_{0}\right)$, it is convenient to change the integration variable in Eq. (24) as

$$
U\left(\ell, t \mid r_{0}\right)=\frac{4}{\pi t} \int_{0}^{\infty} d z z e^{-z^{2}} \operatorname{Re}\left(A\left(z R / \sqrt{D t}, r_{0} / R\right) e^{-B(z R / \sqrt{D t}) \ell / R}\right) .
$$

\subsection{Short-time asymptotic behavior}

The limit $t \rightarrow 0$ corresponds to the large- $z$ expansions of $A(z, r)$ and $B(z)$ :

$$
\begin{aligned}
& A(z, r) \simeq \frac{i}{2 r^{\frac{1}{2}}} e^{-i z(r-1)} \quad(z \rightarrow \infty), \\
& B(z) \simeq \frac{1}{2}+i z+O(1 / z) \quad(z \rightarrow \infty) .
\end{aligned}
$$

Substituting these approximations into Eq. (28), we get

$$
U\left(\ell, t \mid r_{0}\right) \simeq \frac{e^{-\ell /(2 R)}}{\left(r_{0} / R\right)^{\frac{1}{2}}} \frac{\left(r_{0}-R+\ell\right) e^{-\left(r_{0}-R+\ell\right)^{2} /(4 D t)}}{\sqrt{4 \pi D t^{3}}} \quad(t \rightarrow 0) .
$$

Apart from the factor $e^{-\ell /(2 R)}\left(r_{0} / R\right)^{-\frac{1}{2}}$, this expression is identical to Eq. (4) for diffusion in the half-line, if $x_{0}=r_{0}-R$ denotes the distance to the boundary. This is not surprising because the circle looks locally flat at short times, and the behavior should be close to that of the half-plane. In turn, the supplementary factor $e^{-\ell /(2 R)}\left(r_{0} / R\right)^{-\frac{1}{2}}$ accounts for the curvature of the boundary. Note that in the case $r_{0}=R$, the same result could alternatively be derived by analyzing Eq. (C.1) in the short-time limit (i.e., $p \rightarrow \infty)$.

In the limit $t \rightarrow 0$, the particle does not spend enough time near the boundary to allow for the boundary local time $\ell_{t}$ to cross a given threshold $\ell$, and the probability density of too short first-crossing times, $t \ll \ell^{2} /(4 D)$, is extremely small, even if the particle starts on the boundary. At first thought, this statement may sound to

contradict the well-known property that (reflected) Brownian motion that crossed a smooth boundary returns infinitely many times to that boundary during an infinitely short period [31]. This seeming contradiction reflects the crucial difference between the 
Statistics of boundary encounters...

boundary local time, $\ell_{t}$, and the number of encounters $N_{t}^{a}$ with a thin boundary layer of width $a$ (i.e., the number of returns up to $t$ ). The latter is defined for any $a>0$ but diverges in the limit $a \rightarrow 0$. This divergence, $N_{t}^{a} \rightarrow N_{t}^{0}=+\infty$, is evoked in the above statement about infinitely many returns. In turn, the boundary local time $\ell_{t}$, which is obtained by rescaling $N_{t}^{a}$ by $a$ in Eq. (2), remains a meaningful characteristics of the encounters with the boundary in the limit $a \rightarrow 0$.

\subsection{Long-time asymptotic behavior}

The large- $t$ limit corresponds to the small- $z$ behavior of $A(z, r)$ and $B(z)$ :

$$
\begin{aligned}
& A(z, r) \simeq \frac{i}{2}(1-B(z) \ln r) \quad(z \rightarrow 0), \\
& B(z) \simeq \frac{1}{\ln (1 / z)+\ln 2-\gamma-\frac{\pi i}{2}} \quad(z \rightarrow 0),
\end{aligned}
$$

where $\gamma \approx 0.5772 \ldots$ is the Euler constant. We substitute these expressions in Eq. (28) and then neglect $\ln z$ as a slowly varying function to get

$$
\begin{aligned}
U\left(\ell, t \mid r_{0}\right) & \simeq \frac{1}{\pi t} \exp \left(-\frac{(\ell / R) b_{t}}{b_{t}^{2}+(\pi / 2)^{2}}\right)\left[\sin \left(\frac{(\ell / R) \pi / 2}{b_{t}^{2}+(\pi / 2)^{2}}\right)\left(1-\frac{b_{t} \ln \left(r_{0} / R\right)}{b_{t}^{2}+(\pi / 2)^{2}}\right)\right. \\
& \left.+\cos \left(\frac{(\ell / R) \pi / 2}{b_{t}^{2}+(\pi / 2)^{2}}\right) \frac{(\pi / 2) \ln \left(r_{0} / R\right)}{b_{t}^{2}+(\pi / 2)^{2}}\right],
\end{aligned}
$$

where the remaining integral over $z$ gave the factor $1 / 2$, and we set $b_{t}=\ln (\sqrt{4 D t} / R)-\gamma$. When $b_{t} \gg \pi / 2$ and $b_{t} \gg \sqrt{\ell / R}$, we finally get

$$
U\left(\ell, t \mid r_{0}\right) \simeq 2 \frac{\ell / R+\ln \left(r_{0} / R\right)}{t\left[\ln \left(D t / R^{2}\right)\right]^{2}} \quad(t \rightarrow \infty) .
$$

For comparison, we also present the long-time asymptotic behavior of the probability density $H_{q}\left(t \mid r_{0}\right)$ of the first-passage time to a partially reactive target, which was derived in [29]:

$$
H_{q}\left(t \mid r_{0}\right) \simeq \frac{2\left(\frac{1}{q R}+\ln \left(r_{0} / R\right)\right)}{t\left(\pi^{2}+\left[\ln \left(R^{2} /(4 D t)\right)+2 \gamma-\frac{2}{q R}\right]^{2}\right)} .
$$

In the very long time limit, one gets

$$
H_{q}\left(t \mid r_{0}\right) \simeq 2 \frac{\frac{1}{q R}+\ln \left(r_{0} / R\right)}{t\left[\ln \left(D t / R^{2}\right)\right]^{2}} .
$$

In the particular case $q=\infty$, this behavior coincides with Eq. (35) at $\ell=0$, in agreement with Eq. (25). More generally, both densities $U\left(\ell, t \mid r_{0}\right)$ and $H_{q}\left(t \mid r_{0}\right)$ that are related by Eq. (16), exhibit the same dependence on time in the limit $t \rightarrow 0$. Curiously, the relation (16) is satisfied even for the asymptotic forms in Eqs. (35, 37).

One can see that the probability density $U\left(\ell, t \mid r_{0}\right)$ exhibits a very heavy tail at large t. In particular, any positive moment of the first-crossing time, $\mathbb{E}_{\boldsymbol{x}_{0}}\left\{\mathcal{T}_{\ell}^{k}\right\}$, with $k \in \mathbb{R}_{+}$, is infinite. This divergence is caused by the contribution of very long trajectories that 
explore the plane and can move far away from the target but unavoidably return to it due to the recurrent character of planar diffusion. The same mechanism leads to the infinite mean first-crossing time for diffusion in the half-line. However, the $t^{-3 / 2}$ decay of $U\left(\ell, t \mid x_{0}\right)$ in Eq. (4) ensures that at least the moments $\mathbb{E}_{x_{0}}\left\{\mathcal{T}_{\ell}^{k}\right\}$ with $k<1 / 2$ exist in the one-dimensional setting.

Figure 2 illustrates the behavior of the probability density $U(\ell, t \mid R)$ for several values of the threshold $\ell$. For cross-validation, this density was computed by two independent methods: (i) the numerical evaluation of the integral in Eq. (27), and (ii) the numerical computation of the inverse Laplace transform in Eq. (C.4) by the Talbot algorithm. Both methods show an excellent agreement (circles versus crosses). At $\ell / R=0.1$ and $\ell / R=1$, the short-time asymptotic relation (31) accurately captures the behavior of $U(\ell, t \mid R)$ at short and even moderate times, $t \lesssim R^{2} / D$, but fails at long times, as expected. In particular, one can use the simple form of Eq. (31) to estimate the most probable first-crossing time: $t_{\mathrm{mp}} \simeq\left(r_{0}-R+\ell\right)^{2} /(6 D)$. As the mean value is infinite, the most probable time plays the role of a natural time scale. At long times, $t \gg R^{2} / D$, Eq. (35) correctly captures the long-time behavior but approaches it slowly, particularly for large $\ell$. In contrast, the approximate relation (34) turns out to be surprisingly accurate for large and even intermediate times.

The quality of both asymptotic relations $(31,35)$ is much lower in the case $\ell / R=10$ (Fig. 2(c)). On one hand, Eq. (31) does not reproduce correctly the maximum of $U(\ell, t \mid R)$ and captures only the steep decay at small $t$. In other words, in the range of validity of this relation (i.e., $t \lesssim R^{2} / D$ ), the density $U(\ell, t \mid R)$ is already very small and thus not much useful in practice. On the other hand, the approach to Eq. (35) is very slow so that this asymptotic relation becomes accurate only at extremely large times. In contrast, one can still rely on the approximate Eq. (34).

\subsection{Exploration size of trajectories}

In order to quantify the relative contributions of trajectories with different spatial extents into the heavy tail of the probability density $U(\ell, t \mid R)$, we introduce an absorbing circle of radius $L>R$ and compute the probability density of the first-crossing time $\mathcal{T}_{\ell}$ for the trajectories that remained inside that circle up to $\mathcal{T}_{\ell}$. In order words, while computing the statistics of the first-crossing times, we discard all trajectories that hit the circle of radius $L$ up to time $\mathcal{T}_{\ell}$. In Appendix D, we describe an extension of the spectral approach from $[18,23-25]$ to compute the density $U_{L}\left(\ell, t \mid r_{0}\right)$ in the presence of the absorbing circle. Expectedly, the density $U_{L}\left(\ell, t \mid r_{0}\right)$ is not normalized to 1 , and

$1-\int_{0}^{\infty} d t U_{L}\left(\ell, t \mid r_{0}\right)$ is the probability that the trajectory $\boldsymbol{X}_{t}$ has crossed the circle of radius $L$ before the associated boundary local time could reach the level $\ell$. In this setting, we could not derive a fully explicit representation for $U_{L}\left(\ell, t \mid r_{0}\right)$ and thus performed the Laplace transform inversion in Eq. (D.3) numerically by the Talbot algorithm.

Figure 3 shows the probability densities $U_{L}(\ell, t \mid R)$ for several values of $L$ (with $L=\infty$ corresponding to the former case without absorbing circle). As the "restricted" 

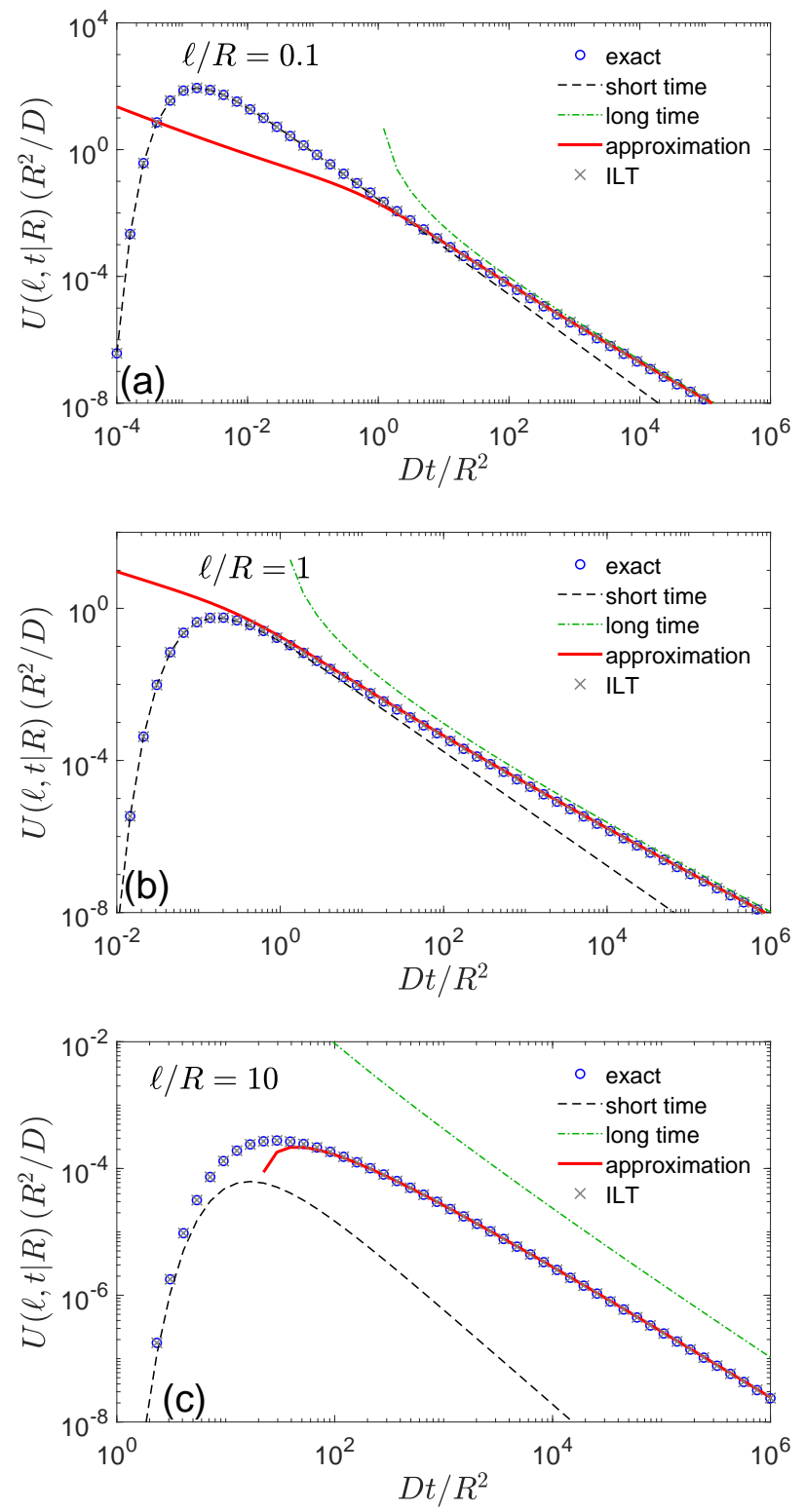

Figure 2. The probability density $U(\ell, t \mid R)$ as a function of $t$ for diffusion outside the disk of radius $R=1$, with $D=1, \ell=0.1$ (a), $\ell=1$ (b), and $\ell=10$ (c). Empty circles show the exact solution obtained by numerical computation of the integral in Eq. (27); dashed line presents the short-time asymptotic behavior (31); solid and dash-dotted lines show the long-time asymptotic relations (34) and (35), respectively; crosses present the numerical computation of the inverse Laplace transform in Eq. (C.4) by the Talbot algorithm. 

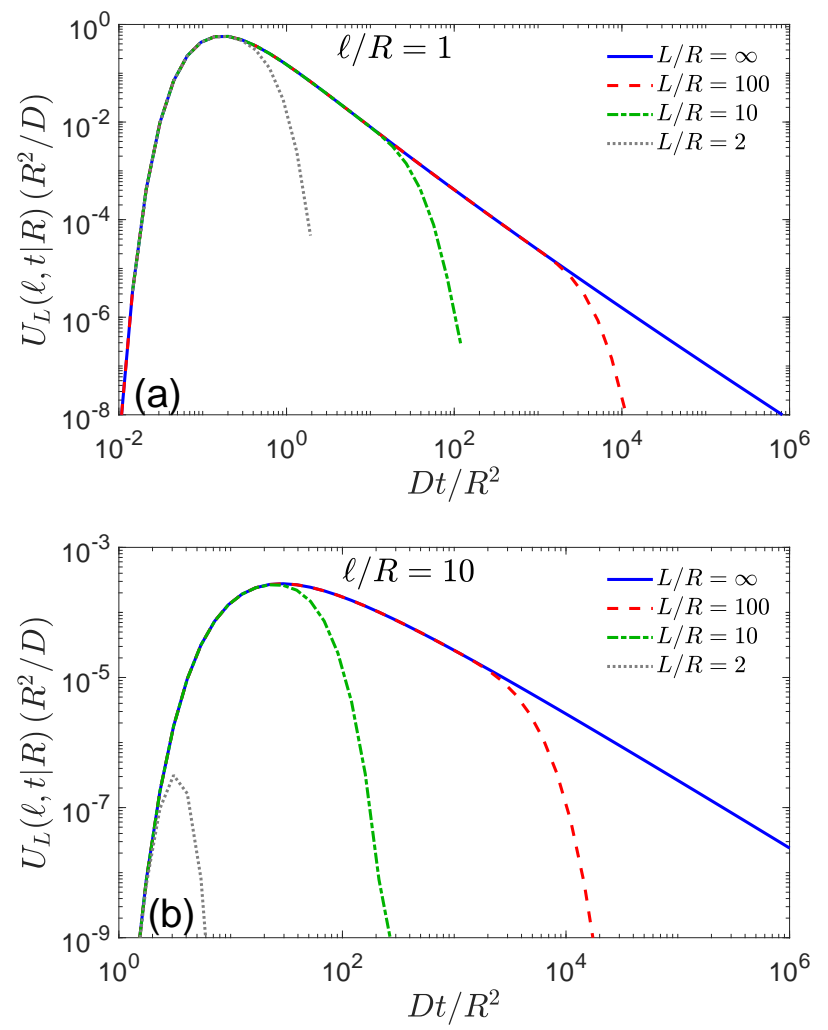

Figure 3. The probability density $U(\ell, t \mid R)$ (solid line) as a function of $t$ for diffusion outside the disk of radius $R=1$, with $D=1, \ell=1$ (a) and $\ell=10$ (b). This density is compared to the probability densities $U_{L}(\ell, t \mid R)$ in the presence of the absorbing circle of radius $L$, with three values of $L$ as indicated in the plot. All these densities were obtained via the numerical computation of the inverse Laplace transform in Eq. (D.3) by the Talbot algorithm. Note that two last curves in the panel (a) were truncated at large times to avoid numerical instabilities of the Laplace transform inversion.

trajectories explore a bounded domain between two concentric circles (of radii $R$ and $L$ ), the particle returns to the inner circle (target) more often, and the boundary local time increases faster. As a consequence, the probability density $U_{L}\left(\ell, t \mid r_{0}\right)$ of the associated first-crossing time is expected to decay much faster as $t \rightarrow \infty$, as confirmed by Fig. 3. This faster decay is also related to the fact that keeping long trajectory within a bounded region becomes more and more unlikely as time goes on. This figure explicitly illustrates that the heavy tail of the density $U(\ell, t \mid R)$ in Eq. (35) is caused by very long trajectories that explore the unbounded planar space and thus create long stalling periods, during which the boundary local time does not change.

\section{Discussion and conclusion}

In this paper, we considered diffusion in the exterior of a disk and derived the explicit integral representations (21) and (24) for the probability densities $\rho\left(\ell, t \mid r_{0}\right)$ and $U\left(\ell, t \mid r_{0}\right)$ of the boundary local time $\ell_{t}$ and of the first-crossing time $\mathcal{T}_{\ell}$, respectively. As the former 
density $\rho\left(\ell, t \mid r_{0}\right)$ was investigated in [23], we mainly focused on the latter one. With the aid of the integral representation (24), we deduced Eqs. (31, 35) describing the short-time and the long-time asymptotic behavior of $U\left(\ell, t \mid r_{0}\right)$, respectively. Moreover, Eq. (34) was checked to be an accurate approximation of $U\left(\ell, t \mid r_{0}\right)$ for moderate and long times. We illustrated that the probability density $U\left(\ell, t \mid r_{0}\right)$ vanishes at short times, reaches the maximum at intermediate times $\sim \frac{1}{D}\left(r_{0}-R+\ell\right)^{2}$, and exhibits a heavy long-time tail such that all positive-order moments are infinite. To quantify the effect of long excursions between encounters, we also analyzed the probability density $U_{L}\left(\ell, t \mid r_{0}\right)$ in the presence of an absorbing circle of radius $L$.

As discussed in Secs. 1 and 2, the boundary local time $\ell_{t}$ is a proxy of the number of encounters, $\mathcal{N}_{t}^{a}$, of a diffusing particle with the boundary layer $\Gamma_{a}$ of width $a$ up to time $t$ (here, $\Gamma=\partial \Omega$ ). Consequently, the first-crossing time $\mathcal{T}_{\ell}$ describes the moment when the number of encounters exceeds a prescribe threshold. While the random variables $\ell_{t}$ and $\mathcal{T}_{\ell}$ characterize the reflecting boundary, they form a natural ground for incorporating surface reactivity and to describe various diffusion-mediated surface phenomena such as diffusion-influenced chemical reactions, permeation across biological membranes, surface relaxation in nuclear magnetic resonance, etc. [18, 19]. For instance, $U\left(0, t \mid r_{0}\right)=H_{\infty}\left(t \mid r_{0}\right)$ describes the first-passage time to the perfectly reactive boundary [32], whereas $H_{q}\left(t \mid r_{0}\right)$, expressed through Eq. (16) in terms of $U\left(\ell, t \mid r_{0}\right)$, determines the reaction time on a partially reactive boundary [33-49]. More sophisticated surface reaction mechanisms can also be implemented with the help of $U\left(\ell, t \mid r_{0}\right)$, as discussed in [18].

Interestingly, the probability densities $\rho\left(\ell, t \mid r_{0}\right)$ and $U\left(\ell, t \mid r_{0}\right)$ also describe the first-encounter statistics of two independent particles diffusing in the plane. In fact, associating the coordinate frame with one of the particles, the diffusive dynamics of two circular particles of radii $R_{1}$ and $R_{2}$ with diffusivities $D_{1}$ and $D_{2}$ can be mapped onto planar diffusion of a single point-like particle with diffusivity $D=D_{1}+D_{2}$ toward a static circular target of radius $R=R_{1}+R_{2}$. As a consequence, $\ell_{t} / a$ is the number of encounters of such two particles, where $a$ is the separation between two particles at each encounter. In other words, a single encounter is counted when two particles hit each other and then moved away and become separated by a distance exceeding $a$. In turn, $\mathcal{T}_{a N}$ is the first moment when the number of such encounters exceeds $N$. As an encounter of two species is the necessary step for most bimolecular reactions, reproduction in animals, and virus spreading in humans, both quantities can be used in describing various encounters in chemistry, biology and ecology (see [50-58] and references therein).

Even though the explicit representations (21) and (24) were derived for the particular case of a disk, the asymptotic behavior of the probability density $U\left(\ell, t \mid \boldsymbol{x}_{0}\right)$ is expected to remain valid for diffusion in the exterior of a general compact planar domain with smooth boundary. Indeed, at short times, $t \ll R_{c}^{2} / D$, the boundary looks locally flat with respect to the radius of curvature $R_{c}$ of the boundary in a vicinity of the starting point. As a consequence, the time dependence of the density $U\left(\ell, t \mid \boldsymbol{x}_{0}\right)$ is expected to be given by Eq. (4) for the one-dimensional setting, where $x_{0}=\left|\boldsymbol{x}_{0}-\Gamma\right|$ is the 
distance between the starting point $\boldsymbol{x}_{0}$ and the target $\Gamma$. In turn, the correction factor $e^{-\ell /(2 R)}\left(r_{0} / R\right)^{-\frac{1}{2}}$ from Eq. (31) is specific to the disk. Finding the shape-dependent correction factor for other domains presents an interesting perspective. Similarly, at large times, long excursions between encounters are expected to lead to a heavy tail of the probability density $U\left(\ell, t \mid \boldsymbol{x}_{0}\right)$ as $t \rightarrow \infty$. The characteristic $1 /\left(t \ln ^{2}(t)\right)$ decay may remain universal but the dependence on $\ell$ and $\boldsymbol{x}_{0}$ could be shape-specific. Validation and specification of these conjectures present another exciting direction for future research. This problem is tightly related to the asymptotic behavior of the spectrum of the Dirichlet-to-Neumann operator associated with the modified Helmholtz equation [23] (see also Appendix D).

\section{Acknowledgments}

The author acknowledges a partial financial support from the Alexander von Humboldt Foundation through a Bessel Research Award.

\section{Appendix A. Long-time behavior of the mean boundary local time}

According to Eq. (21), the probability density $\rho\left(\ell, t \mid r_{0}\right)$ exhibits a rapid decay for large $\ell$, ensuring the existence of all positive-order moments of the boundary local time $\ell_{t}$ :

$$
\mathbb{E}_{\boldsymbol{x}_{0}}\left\{\ell_{t}^{k}\right\}=\int_{0}^{\infty} d \ell \ell^{k} \rho\left(\ell, t \mid \boldsymbol{x}_{0}\right) \quad(k>0) .
$$

The explicit representation (21) can be used to compute these moments. However, the direct exchange of the order of integrals over $\ell$ in Eq. (A.1) and over $z$ in Eq. (21) is not possible as the resulting integral over $z$ would be divergent. To overcome this limitation, one can first express the time derivative of the moment $\mathbb{E}_{\boldsymbol{x}_{0}}\left\{\ell_{t}^{k}\right\}$ as

$$
\partial_{t} \mathbb{E}_{\boldsymbol{x}_{0}}\left\{\ell_{t}^{k}\right\}=\frac{4 D R^{k-2} \Gamma(k+1)}{\pi} \int_{0}^{\infty} d z z e^{-z^{2} D t / R^{2}} \operatorname{Re}\left(\frac{A\left(z, r_{0} / R\right)}{[B(z)]^{k}}\right) .
$$

Rescaling the integral variable yields

$$
\partial_{t} \mathbb{E}_{\boldsymbol{x}_{0}}\left\{\ell_{t}^{k}\right\}=\frac{4 R^{k} \Gamma(k+1)}{\pi t} \int_{0}^{\infty} d z z e^{-z^{2}} \operatorname{Re}\left(\frac{A\left(z R / \sqrt{D t}, r_{0} / R\right)}{[B(z R / \sqrt{D t})]^{k}}\right) .
$$

In the long-time limit, one uses the small- $z$ expansions $(33,32)$ to get

$$
\begin{aligned}
\partial_{t} \mathbb{E}_{\boldsymbol{x}_{0}}\left\{\ell_{t}^{k}\right\} & \simeq \frac{2 R^{k} \Gamma(k+1)}{\pi t} \int_{0}^{\infty} d z z e^{-z^{2}} \operatorname{Re}\left(i \left[\left(b_{t}-\ln z-\pi i / 2\right)^{k}\right.\right. \\
& \left.\left.-\ln \left(r_{0} / R\right)\left(b_{t}-\ln z-\pi i / 2\right)^{k-1}\right]\right),
\end{aligned}
$$


where $b_{t}=\ln (\sqrt{4 D t} / R)-\gamma$. Integrating this expression over $t$, we get

$$
\begin{aligned}
\mathbb{E}_{\boldsymbol{x}_{0}}\left\{\ell_{t}^{k}\right\} & \simeq \frac{2 R^{k} \Gamma(k+1)}{\pi} \int_{0}^{\infty} d z e^{-z} \operatorname{Im}\left(-\frac{\left(b_{t}-\frac{1}{2} \ln z-\frac{1}{2} \pi i\right)^{k+1}}{k+1}\right. \\
& \left.+\ln \left(r_{0} / R\right) \frac{\left(b_{t}-\frac{1}{2} \ln z-\frac{1}{2} \pi i\right)^{k}}{k}\right),
\end{aligned}
$$

where we also changed the integration variable $z^{2} \rightarrow z$. Using the binomial expansion, one can evaluate these integrals term by term. In particular, we get the mean and the variance in the long-time limit:

$$
\begin{aligned}
\mathbb{E}_{\boldsymbol{x}_{0}}\left\{\ell_{t}\right\} & \simeq R\left(\ln \left(\sqrt{4 D t} / r_{0}\right)-\gamma / 2\right) \\
\operatorname{var}_{\boldsymbol{x}_{0}}\left\{\ell_{t}\right\} & =\mathbb{E}_{\boldsymbol{x}_{0}}\left\{\ell_{t}^{2}\right\}-\left(\mathbb{E}_{\boldsymbol{x}_{0}}\left\{\ell_{t}\right\}\right)^{2} \\
& \simeq R^{2}\left((\ln (\sqrt{4 D t} / R)-\gamma / 2)^{2}-\ln ^{2}\left(r_{0} / R\right)-\frac{\pi^{2}}{12}\right) .
\end{aligned}
$$

These expressions generalize the former results from [23] to an arbitrary starting point.

\section{Appendix B. Numerical computation}

Using Eqs. (33, 32), one can easily check that the integrals in Eqs. (21, 23) converge logarithmically slowly near $z=0$. To improve the accuracy of numerical computations, one can choose an appropriate $\varepsilon \ll 1$ and split the integral into two parts, from 0 to $\varepsilon$, and from $\varepsilon$ to $\infty$. The second integral is then evaluated numerically, whereas the contribution of the first integral, $I_{\varepsilon}$, can be found approximately.

Let us first consider the computation of $Q\left(\ell, t \mid r_{0}\right)$ in Eq. (23), for which

$$
\begin{aligned}
I_{\varepsilon} & =\frac{4}{\pi} \int_{0}^{\varepsilon} \frac{d z}{z} e^{-z^{2} D t / R^{2}} \operatorname{Re}\left(A(z, r) e^{-B(z) \ell / R}\right) \\
& \approx \frac{4}{\pi} \int_{0}^{\varepsilon} \frac{d z}{z} \operatorname{Re}\left(\frac{i}{2}(1-B(z) \ln r) e^{-B(z) \ell / R}\right) \\
& \approx-\frac{2}{\pi} \int_{\ln (1 / \varepsilon)}^{\infty} d y \operatorname{Im}\left(\left(1-\frac{\ln r}{y+b}\right) \exp \left(-\frac{c}{y+b}\right)\right),
\end{aligned}
$$

where $c=\ell / R$ and $b=\ln 2-\gamma-\frac{1}{2} \pi i$. Here we approximated $e^{-z^{2} D t / R^{2}} \approx 1$ for $0 \leq z \leq \varepsilon$, and made the change of variables: $y=\ln (1 / z)$. Note that the above approximation naturally imposes the constraint on $\varepsilon$ : $\varepsilon^{2} \ll R^{2} /(D t)$. In particular, as $t$ increases, one has to choose smaller and smaller $\varepsilon$.

The last integral can be evaluated as

$$
I_{\varepsilon} \approx 1+\frac{2}{\pi} \operatorname{Im}\left(b_{\varepsilon} e^{-c / b_{\varepsilon}}-\left(c+\ln \left(r_{0} / R\right)\right) \operatorname{Ei}_{1}\left(c / b_{\varepsilon}\right)\right),
$$


Statistics of boundary encounters...

where $\operatorname{Ei}_{1}(z)=\int_{1}^{\infty} d k e^{-z k} / k=\Gamma(0, z)$ is the exponential integral, and $b_{\varepsilon}=b+\ln (1 / \varepsilon)$. As a consequence, we get

$$
Q\left(\ell, t \mid r_{0}\right)=1-I_{\varepsilon}-\frac{4}{\pi} \int_{\varepsilon}^{\infty} \frac{d z}{z} e^{-z^{2} D t / R^{2}} \operatorname{Re}\left(A\left(z, r_{0} / R\right) e^{-B(z) \ell / R}\right) .
$$

A similar correction can be used for an accurate computation of $\rho\left(\ell, t \mid r_{0}\right)$ :

$$
\begin{aligned}
\rho\left(\ell, t \mid r_{0}\right) & =S_{\infty}\left(t \mid r_{0}\right) \delta(\ell)-I_{\varepsilon}^{(\rho)} \\
& -\frac{4}{\pi R} \int_{\varepsilon}^{\infty} \frac{d z}{z} e^{-z^{2} D t / R^{2}} \operatorname{Re}\left(A\left(z, r_{0} / R\right) B(z) e^{-B(z) \ell / R}\right),
\end{aligned}
$$

with

$$
\begin{aligned}
I_{\varepsilon}^{(\rho)} & =\frac{4}{\pi R} \int_{0}^{\varepsilon} \frac{d z}{z} e^{-z^{2} D t / R^{2}} \operatorname{Re}\left(A\left(z, r_{0} / R\right) B(z) e^{-B(z) \ell / R}\right) \\
& \approx \frac{2}{\pi R} \operatorname{Im}\left(\operatorname{Ei}_{1}\left(c / b_{\varepsilon}\right)-\frac{\ln \left(r_{0} / R\right)}{c} e^{-c / b_{\varepsilon}}\right) .
\end{aligned}
$$

Note that there is no need for such a correction for computing $U\left(\ell, t \mid r_{0}\right)$ because $I_{\varepsilon}$ does not depend on time and thus disappears after differentiating $Q\left(\ell, t \mid r_{0}\right)$ with respect to $t$.

\section{Appendix C. Alternative representation}

In $[18,23]$, alternative representations for the probability densities $\rho\left(\ell, t \mid \boldsymbol{x}_{0}\right)$ and $U\left(\ell, t \mid \boldsymbol{x}_{0}\right)$ were developed in terms of the eigenmodes of the Dirichlet-to-Neumann operator $\mathcal{M}_{p}$ for an arbitrary Euclidean domain $\Omega$ with a smooth bounded boundary $\partial \Omega$. For a given function $f$ on $\partial \Omega$, the operator $\mathcal{M}_{p}$ associates another function $g=\left.\left(\partial_{n} w\right)\right|_{\partial \Omega}$ on that boundary, where $w$ satisfies the modified Helmholtz equation $(p-D \Delta) w=0$ in $\Omega$ with Dirichlet boundary condition $\left.w\right|_{\partial \Omega}=f$ [59-63]. For instance, for diffusion outside the disk of radius $R$, the probability $Q(\ell, t \mid R)$ reads

$$
Q(\ell, t \mid R)=\mathcal{L}_{p, t}^{-1}\left\{\frac{1}{p} \exp \left(-\mu_{0}^{(p)} \ell\right)\right\},
$$

where $\mathcal{L}_{p, t}^{-1}$ is the inverse Laplace transform with respect to $p$,

$$
\mu_{0}^{(p)}=\sqrt{p / D} \frac{K_{1}(R \sqrt{p / D})}{K_{0}(R \sqrt{p / D})}
$$

is the smallest eigenvalue of the Dirichlet-to-Neumann operator $\mathcal{M}_{p}$ in the exterior of the disk, and $K_{\nu}(z)$ is the modified Bessel function of the second kind [23]. The densities $\rho(\ell, t \mid R)$ and $U(\ell, t \mid R)$ follow immediately by taking the derivatives with respect to $\ell$ and $t$, respectively:

$$
\rho(\ell, t \mid R)=\mathcal{L}_{p, t}^{-1}\left\{\frac{\mu_{0}^{(p)}}{p} \exp \left(-\mu_{0}^{(p)} \ell\right)\right\} .
$$


and

$$
U(\ell, t \mid R)=\mathcal{L}_{p, t}^{-1}\left\{\exp \left(-\mu_{0}^{(p)} \ell\right)\right\}
$$

In this Appendix, we briefly describe a direct derivation of Eq. (C.4) that illustrates the meaning of the boundary local time as a proxy for the number of encounters. Let us consider a regularized version of the problem, in which the particle after hitting the circle at some point $(R, \varphi)$ (in polar coordinates) is released from a bulk point $(R+a, \varphi)$, a distance $a$ above the circle (Fig. 1). The first-crossing time $\mathcal{T}_{\ell}$ can then be represented as $\mathcal{T}_{\ell}=\tau_{1}+\tau_{2}+\ldots+\tau_{N}$, where $\tau_{i}$ is the duration of bulk diffusion after $i$-th release (here we assumed that the particle starts on the circle), and $N \approx \ell / a$ is the number of such bulk explorations, see Eq. (2). The random variables $\tau_{i}$ are independent and identically distributed, with a well-known moment-generating function [26]:

$$
\mathbb{E}\left\{e^{-p \tau_{i}}\right\}=\frac{K_{0}((R+a) \sqrt{p / D})}{K_{0}(R \sqrt{p / D})} .
$$

As a consequence, one has

$$
\begin{aligned}
\mathbb{E}\left\{e^{-p \mathcal{T}_{\ell}}\right\} & =\left(\mathbb{E}\left\{e^{-p \tau_{1}}\right\}\right)^{N} \\
& =\exp \left(N \ln \left(\frac{K_{0}((R+a) \sqrt{p / D})}{K_{0}(R \sqrt{p / D})}\right)\right) \\
& \simeq \exp \left(\frac{\ell}{a} \ln \left(1+\frac{K_{0}^{\prime}(R \sqrt{p / D})}{K_{0}(R \sqrt{p / D})} a \sqrt{p / D}\right)\right) \\
& \rightarrow \exp (-\ell \underbrace{\ell \sqrt{p / D} \frac{K_{1}(R \sqrt{p / D})}{K_{0}(R \sqrt{p / D})}}_{=\mu_{0}^{(p)}}) \quad(a \rightarrow 0) .
\end{aligned}
$$

As a consequence, the inverse Laplace transform of the right-hand side with respect to $p$ yields $U(\ell, t \mid R)$ in Eq. (C.4).

\section{Appendix D. Concentric annulus}

In order to quantify the relative contributions of far-reaching trajectories, it is convenient to introduce an absorbing circle of radius $L$. In other words, we are interested in diffusion inside a circular annulus, $\Omega=\left\{\boldsymbol{x} \in \mathbb{R}^{2}: R<|\boldsymbol{x}|<L\right\}$. In this Appendix, we adopt

the spectral approach from [18,23-25] to deduce the distribution of the boundary local time on the inner circle with the constraint of not hitting the outer circle. For brevity, we skip details and only sketch the main formulas.

Due to rotational invariance, the eigenfunctions of the Dirichlet-to-Neumann operator $\mathcal{M}_{p}$ associated to the inner circle are the Fourier harmonics,

$$
v_{n}^{(p)}=\frac{e^{i n \varphi}}{\sqrt{2 \pi R}} \quad(n \in \mathbb{Z})
$$


whereas the associated eigenvalues are

$$
\mu_{n, L}^{(p)}=-\alpha \frac{K_{n}(\alpha L) I_{n}^{\prime}(\alpha R)-I_{n}(\alpha L) K_{n}^{\prime}(\alpha R)}{K_{n}(\alpha L) I_{n}(\alpha R)-I_{n}(\alpha L) K_{n}(\alpha R)}
$$

with $\alpha=\sqrt{p / D}$ and the prime denoting the derivative with respect to the argument (see similar computations in [23,24]). The decomposition (26) of the first-crossing time $\mathcal{T}_{\ell}$ implies that its probability density is obtained by convolution of two densities, which in the Laplace domain reads

$$
U_{L}\left(\ell, t \mid r_{0}\right)=\mathcal{L}_{p, t}^{-1}\left\{\tilde{H}_{L}\left(p \mid r_{0}\right) \exp \left(-\mu_{0, L}^{(p)} \ell\right)\right\}
$$

Here

$$
\tilde{H}_{L}\left(p \mid r_{0}\right)=\frac{K_{0}(\alpha L) I_{0}\left(\alpha r_{0}\right)-I_{0}(\alpha L) K_{0}\left(\alpha r_{0}\right)}{K_{0}(\alpha L) I_{0}(\alpha R)-I_{0}(\alpha L) K_{0}(\alpha R)}
$$

is the Laplace transform of the probability density of the first-passage time to the inner circle in the presence of an outer absorbing circle (expectedly, this function vanishes on the outer circle, $r_{0}=L$, and is equal to 1 on the inner circle, $r_{0}=R$ ). In turn, $\exp \left(-\mu_{0, L}^{(p)} \ell\right)$ is the Laplace transform of the probability density $U(\ell, t \mid R)$ (compare with Eq. (C.4)). In the limit $L \rightarrow \infty$, the eigenvalue $\mu_{0, L}^{(p)}$ from Eq. (D.2) tends to $\mu_{0}^{(p)}$ from Eq. (C.2), and one retrieves Eq. (C.4). Similarly, one gets

$$
Q_{L}\left(\ell, t \mid r_{0}\right)=\mathcal{L}_{p, t}^{-1}\left\{\tilde{H}_{L}\left(p \mid r_{0}\right) \frac{\exp \left(-\mu_{0, L}^{(p)} \ell\right)}{p}\right\}
$$

and

$$
\rho_{L}\left(\ell, t \mid r_{0}\right)=\mathcal{L}_{p, t}^{-1}\left\{\tilde{H}_{L}\left(p \mid r_{0}\right) \frac{\mu_{0, L}^{(p)}}{p} \exp \left(-\mu_{0, L}^{(p)} \ell\right)\right\} .
$$

To get the long-time behavior, one can evaluate the above expressions in the limit $p \rightarrow 0$; in particular,

$$
\lim _{p \rightarrow 0} \tilde{H}_{L}\left(p \mid r_{0}\right)=\frac{\ln \left(L / r_{0}\right)}{\ln (L / R)}, \quad \lim _{p \rightarrow 0} \mu_{0}^{(p)}=\frac{1}{R \ln (L / R)},
$$

from which

$$
Q_{L}(\ell, \infty \mid R)=\frac{\ln \left(L / r_{0}\right)}{\ln (L / R)} \exp \left(-\frac{\ell / R}{\ln (L / R)}\right) .
$$

This is the probability that the threshold $\ell$ is crossed by the boundary local time $\ell_{t}$ before the trajectory $\boldsymbol{X}_{t}$ hit the outer circle of radius $L$. As $L \rightarrow \infty$, this probability tends to 1 , but this approach is slow and controlled by $\ln (L / R)$.

[1] Lévy P 1965 Processus Stochastiques et Mouvement Brownien (Paris: Gauthier-Villard).

[2] Ito K and McKean HP 1965 Diffusion Processes and Their Sample Paths (Berlin: Springer-Verlag).

[3] Freidlin M 1985 Functional Integration and Partial Differential Equations (Annals of Mathematics Studies, New Jersey: Princeton University Press, Princeton).

[4] Darling DA and Kac M 1957, Trans. Am. Math. Soc. 84 444-458.

[5] Ray D 1963 Illinois J. Math. 7 615-630. 
[6] Knight FB 1963 Trans. Amer. Math. Soc. 109 56-86.

[7] Agmon N 1984 J. Chem. Phys. 81 3644-3647.

[8] Berezhkovskii AM, Zaloj V, and Agmon N 1998 Phys. Rev. E 57 3937-3947.

[9] Dhar A and Majumdar SN 1999 Phys. Rev. E 59 6413-6418.

[10] Yuste SB and Acedo L 2001 Phys. Rev. E 64061107.

[11] Godrèche C and Luck JM 2001, J. Stat. Phys. 104 489-524.

[12] Majumdar SN and Comtet A 2002 Phys. Rev. Lett. 89060601.

[13] Bénichou O, Coppey M, Klafter J, Moreau M, and Oshanin G 2003 J. Phys. A.: Math. Gen. 36 7225-7231.

[14] Condamin S, Bénichou O, and Moreau M 2005 Phys. Rev. E 72016127.

[15] Condamin S, Tejedor V, and Bénichou O 2007 Phys. Rev. E 76 050102R.

[16] Burov S and Barkai E 2007 Phys. Rev. Lett. 98250601.

[17] Burov S and Barkai E 2011 Phys. Rev. Lett. 107170601.

[18] Grebenkov DS 2020 Phys. Rev. Lett. 125078102.

[19] Grebenkov DS 2007 Phys. Rev. E 76041139.

[20] Saisho Y 1987 Probab. Theory Rel. Fields 74 455-477.

[21] Papanicolaou VG 1990 Probab. Theory Rel. Fields 87 27-77.

[22] Bass RF, Burdzy K, and Chen ZQ 2008 Proc. London Math. Soc. 96 273-311.

[23] Grebenkov DS 2019 Phys. Rev. E 100062110.

[24] Grebenkov DS 2020 Phys. Rev. E 102032125.

[25] Grebenkov DS 2020 J. Stat. Mech. 103205.

[26] Redner S 2001 A Guide to First Passage Processes (Cambridge: Cambridge University press).

[27] Epstein CL and Schotland J 2008 SIAM Rev. 50 504-520.

[28] Carslaw HS and Jaeger JC 1959 Conduction of Heat in Solids, 2nd Ed. (Oxford: Clarendon).

[29] Grebenkov DS, Metzler R, and Oshanin G 2018 Phys. Chem. Chem. Phys. 20 16393-16401.

[30] Levitz PE, Zinsmeister M, Davidson P, Constantin D, and Poncelet O 2008, Phys. Rev. E 78 030102(R).

[31] Mörters P and Peres Y 2010 Brownian Motion (Cambridge Series in Statistical and Probabilistic Mathematics, Cambridge: Cambridge University Press).

[32] Smoluchowski M 1917 Z. Phys. Chem. 92U 129-168.

[33] Collins FC and Kimball GE 1949, J. Coll. Sci. 4 425-437.

[34] Sano H and Tachiya M 1979, J. Chem. Phys. 71 1276-1282.

[35] Shoup D and Szabo A 1982 Biophys. J. 40 33-39.

[36] Zwanzig R 1990 Proc. Natl. Acad. Sci. USA 87, 5856-5857.

[37] Sapoval B 1994 Phys. Rev. Lett. 73 3314-3317.

[38] Filoche M and Sapoval B 1999 Eur. Phys. J. B 9 755-763.

[39] Bénichou O, Moreau M, and Oshanin G 2000 Phys. Rev. E 61 3388-3406.

[40] Grebenkov DS, Filoche M, and Sapoval B 2003 Eur. Phys. J. B 36, 221-231.

[41] Berezhkovskii AM, Makhnovskii Y, Monine M, Zitserman V, and Shvartsman S $2004 \mathrm{~J}$. Chem. Phys. 12111390.

[42] Grebenkov DS 2006 Partially Reflected Brownian Motion: A Stochastic Approach to Transport Phenomena, in "Focus on Probability Theory", Ed. L. R. Velle, pp. 135-169 (New York: Nova Science Publishers).

[43] Grebenkov DS, Filoche M, and Sapoval B 2006, Phys. Rev. E 73021103.

[44] Bressloff PC, Earnshaw BA, and Ward MJ 2008, SIAM J. Appl. Math. 68 1223-1246.

[45] Reingruber J and Holcman D 2009 Phys. Rev. Lett. 103148102.

[46] Lawley SD and Keener JP 2015 SIAM J. Appl. Dyn. Sys. 14 1845-1867.

[47] Grebenkov DS and Oshanin G 2017 Phys. Chem. Chem. Phys. 19 2723-2739.

[48] Bernoff A, Lindsay A, and Schmidt D 2018 Multiscale Model. Simul. 16 1411-1447.

[49] Grebenkov DS 2019 J. Chem. Phys. 151104108.

[50] Tejedor V, Schad M, Bénichou O, Voituriez R, and Metzler R 2011 J. Phys. A.: Math. Theor. 44 
395005.

[51] Amitai A, Kupka I, and Holcman D 2012 Phys. Rev. Lett. 109108302.

[52] Bressloff PC and Newby J 2013 Rev. Mod. Phys. 85 135-196.

[53] Giuggioli L, Pérez-Becker S, and Sanders DP 2013, Phys. Rev. Lett. 110058103.

[54] Tzou JC, Xie S, and Kokolnikov T 2014, Phys. Rev. E 90062138.

[55] Agliari E, Blumen A, and Cassi D 2014 Phys. Rev. E 89052147.

[56] Agliari E, Cassi D, Cattivelli L, and Sartori F 2016, Phys. Rev. E 93052111.

[57] Peng J and Agliari E 2019 Phys. Rev. E 100062310.

[58] Le Vot F, Yuste SB, Abad E, and Grebenkov DS 2020 Phys. Rev. E 102032118.

[59] Arendt W, ter Elst AFM, Kennedy JB, and Sauter M 2014 J. Funct. Anal. 266 1757-1786.

[60] Daners D 2014 Positivity 18 235-256.

[61] Arendt W and ter Elst AFM 2015 Potential Anal. 43 313-340.

[62] Hassell A and Ivrii V 2017 J. Spectr. Theory 7 881-905.

[63] Girouard A and Polterovich I 2017 J. Spectr. Theory 7 321-359. 